(C) The Authors 2018. This is an Open Access article, distributed under the terms of the Creative Commons

Attribution licence (http://creativecommons.org/licenses/by/4.0/), which permits unrestricted re-use,

distribution, and reproduction in any medium, provided the original work is properly cited.

\title{
Environmental enteric dysfunction and systemic inflammation predict reduced weight but not length gain in rural Bangladeshi children
}

\author{
Rebecca K. Campbell ${ }^{1}$, Kerry J. Schulze ${ }^{1 *}$, Saijuddin Shaikh ${ }^{1,2}$, Rubhana Raqib ${ }^{3}$, Lee S. F. Wu ${ }^{1}$, \\ Hasmot Ali ${ }^{1,2}$, Sucheta Mehra ${ }^{1}$, Keith P. West $\mathrm{Jr}^{1}$ and Parul Christian ${ }^{1}$ \\ ${ }^{1}$ Department of International Health, Center for Human Nutrition, Johns Hopkins Bloomberg School of Public Health, \\ Baltimore, MD 21205, USA \\ ${ }^{2}$ IiVitA Project, Gaibandha, Bangladesh \\ ${ }^{3}$ icddr, b, Mohakhali, Dhaka 1212, Bangladesh
}

(Submitted 24 July 2017 - Final revision received 14 November 2017 - Accepted 6 December 2017)

\section{Abstract}

Environmental enteric dysfunction (EED) and systemic inflammation (SI) are common in developing countries and may cause stunting. In Bangladesh, $>40 \%$ of preschool children are stunted, but EED and SI contributions are unknown. We aimed to determine the impact of EED and SI (assessed with multiple indicators) on growth in children ( $n$ 539) enrolled in a community-based randomised food supplementation trial in rural Bangladesh. EED was defined with faecal myeloperoxidase, $\alpha-1$ antitrypsin and neopterin and serum endotoxin core antibody and glucagon-like peptide-2, consolidated into gut inflammation (GI) and permeability (GP) scores, and urinary lactulose:mannitol $\alpha-1$ acid glycoprotein (AGP) characterised SI. Biomarker associations with anthropometry (15-, 18- and 24-month length-for-age (LAZ), weightfor-length (WLZ) and weight-for-age (WAZ) $z$ scores) were examined in pairwise correlations and adjusted mixed-effects regressions. Stunting, wasting and underweight prevalence at 18 months were 45, 15 and 37\%, respectively, with elevated EED and SI markers common. EED and SI were not associated with 15-24-month length trajectory. Elevated (worse) GI and GP scores predicted reduced 18-24-month WLZ change ( $\beta-0.01$ (sE 0.00$) z$ score/month for both). Elevated GP was also associated with reduced 15-18-month WLZ change ( $\beta-0 \cdot 03$ (se $0 \cdot 01$ ) $z$ score/month) and greater 15-month WLZ ( $\beta$ 0.16 (SE 0.05)). Higher AGP was associated with reduced prior and increased subsequent WLZ change ( $\beta-0.04$ (SE 0.01 ) and $\beta 0.02$ (sE 0.00$) z$ score/month for 15-18 and 18-24 months). The hypothesised link from EED to stunting was not observed in this sample of Bangladeshi 18-month-olds, but the effects of EED on constrained weight gain may have consequences for later linear growth or for other health and development outcomes.

Key words: Enteropathy: Intestinal health: Linear growth: Stunting: Early childhood: South Asia

Resolving stunting (length-for-age (LAZ) $<-2 z$ scores below the reference median ${ }^{(1)}$ ) continues to elude public health practitioners, as its risk factors are tightly aligned with poverty but its prevention and remedy are apparently far more complex ${ }^{(2)}$. Stunting is associated with an increased risk of early childhood morbidity and mortality ${ }^{(3)}$ and often persists into adulthood with lifelong and intergenerational consequences for health, human capital and economic development ${ }^{(4,5)}$. Patterns differ somewhat by region, but stunting tends to emerge in the first few months of life and worsen into the 2 nd year before stabilising but typically persisting in the absence of intervention $^{(6)}$. In Bangladesh, the prevalence of stunting has fallen over the past decade, but national estimates continue to place it at over $40 \%$, and the prevalence is even higher among rural populations and those of lowest socio-economic status ${ }^{(7,8)}$.
A pervasive subclinical disease of the small intestine, termed 'environmental enteric dysfunction' or EED, may help explain the burden and persistence of stunting in low- and middle-income countries. EED is thought to result from chronic exposure to environmental pathogens and toxins ${ }^{(9-11)}$, and may lead to impaired growth through malabsorption of nutrients and chronic systemic inflammation (SI) ${ }^{(12,13)}$. Observational studies have reported high prevalence of EED in numerous settings ${ }^{(14-16)}$, but findings are mixed regarding associations between markers of EED and attained length or rate of linear growth ${ }^{(17-23)}$. Further, the extent to which EED contributes to stunting in rural Bangladesh has been explored only minimally in the existing literature.

In a prior study, we reported that lactulose:mannitol ratio (L:M) and other markers of EED and SI were widely elevated in a subset of 18-month-old children completing participation in a

Abbreviations: AGP, $\alpha$-1 acid glycoprotein; CFS, complementary food supplements; CRP, C-reactive protein; EED, environmental enteric dysfunction; EndoCAb, endotoxin core antibody; GI, gut inflammation; GLP-2, glucagon-like peptide-2; GP, gut permeability; LAZ, length-for-age $z$ score; L:M, lactulose: mannitol; SI, systemic inflammation; WAZ, weight-for-age $z$ score; WLZ, weight-for-length $z$ score

* Corresponding author: K. J. Schulze, email kschulz1@jhu.edu 
community-based randomised controlled trial of complementary food supplements (CFS) in rural northwest Bangladesh $^{(24)}$. Despite not seeing effects of supplementation on markers of EED or SI, cross-tabulations with baseline anthropometric measures revealed some unexpected positive associations between 6-month size and subsequent gut dysfunction, suggesting a potential for associations between EED markers and subsequent growth to be confounded by prior anthropometry. These observations highlight a need to investigate EED in relation to growth trajectories using longitudinal models of length and weight that can address this potential confounding. In the present analysis, we examine, in the same study, length and weight and their trajectories from 15-24 months relative to a panel of EED biomarkers to determine the extent to which markers of EED and SI explain variability in children's growth in their 2nd year of life.

\section{Methods}

\section{Setting}

Participants were a subset of children enrolled in a communitybased randomised controlled CFS trial in the Gaibandha and Rangpur districts of northwest Bangladesh ${ }^{(25)}$. The setting is rural and densely populated with agrarian livelihoods and small household landholdings, typical of rural South Asia. The research site has been host to several randomised nutrition and health trials $^{(26-28)}$ and numerous observational studies ${ }^{(29-32)}$ to characterise and improve maternal and child health.

In the CFS trial, child and household socio-demographic characteristics were assessed by interviewer-administered questionnaire at enrolment (age 6 months), and anthropometry including length and weight was assessed by trained and standardised interviewers at ages 6, 9, 12, 15, 18 and 24 months. To ensure the quality of anthropometric measurements, a randomly selected subset of measurements was rechecked by expert anthropometrists and interviewers were retrained and re-standardised as needed.

\section{Environmental enteric dysfunction assessment}

In a geographically designated subset of trial participants, biospecimens were collected at age 18 months to measure EED and SI. A target sample size of 500 balanced across the five study arms was set based on a primary aim of detecting effects of supplementation on L:M, within the logistical constraints of the methodology and setting. The sample size allowed for detection of a difference in L:M of 0.46 between supplemented groups and the control, assuming L:M standard deviation equal to 0.64 based on prior pilot data from the same study area $(\mathrm{K}$ Schulze, unpublished results), $\alpha=0.05, \beta=0.20$ and adjusting for multiple comparisons. Biospecimens were collected between October 2013 and May 2014 to reach the target sample size. Urine following lactulose and mannitol dosing, blood for processing to serum and stool samples were collected. The full details of the sample collection and laboratory analyses have been described previously ${ }^{(24)}$. In brief, urine collected for $2-3 \mathrm{~h}$ following lactulose and mannitol solution dosing was weighed, mixed with chlorhexidine and stored in liquid $\mathrm{N}_{2}$ pending shipment to a collaborating laboratory at icddr,b, where it was analysed by high-pressure ion chromatography (Dionex; Thermo Fisher Scientific) for concentrations of lactulose and mannitol (CV 3.6 and 9.4\%, respectively). Blood collected during the same field clinic visit was allowed to clot and then, in the project laboratory, centrifuged and the serum transferred to cryovials and stored in liquid $\mathrm{N}_{2}$ for shipment to the Center for Human Nutrition laboratory at JHSPH. A single stool sample for each participant was collected in the household using sterilised collection materials, stored and transported in Styrofoam cold boxes and then processed in the project laboratory and stored in liquid $\mathrm{N}_{2}$ for shipment to JHSPH.

Stool samples were analysed for myeloperoxidase (MPO) and neopterin (NEO), markers of immune activity in the wall of the small intestine ${ }^{(33,34)}$, and $\alpha-1$ antitrypsin (AAT), a marker of protein-losing enteropathy ${ }^{(35)}$, using commercially available ELISA kits (MPO and NEO; ALPCO Diagnostics; AAT; BioVendor, LLC). Serum was analysed for IgG endotoxin core antibody (EndoCAb IgG; Hycult Biotech), a marker of intestinal permeability ${ }^{(20)}$, and glucagon-like peptide-2 (GLP-2), a growth factor indicative of enterocyte proliferation and repair ${ }^{(36)}$, as well as C-reactive protein (CRP) and $\alpha-1$ acid glycoprotein (AGP), markers of SI. EndoCAb and GLP-2 were assessed with commercial ELISA kits (EndoCAb IgG and GLP-2; EMD Millipore), whereas CRP was assessed by chemiluminescent immunoassay (Siemens Diagnostics) and AGP by commercial radial immunodiffusion kit (Kent Laboratories). Standards and controls provided in the kits, along with participant samplederived control samples, were run in duplicate on each plate and $\mathrm{CV}$ monitored.

\section{Data management}

Household characteristics assessed at baseline (child age 6 months) included maternal and paternal education and profession, asset ownership and the physical structure of the house. Assets and house structure variables were captured in a wealth score (living standards index (LSI)) according to a method previously developed for this setting ${ }^{(37)}$, and dichotomised as low and high around the median value within this study sample.

Lactulose and mannitol concentrations were converted to the recovery ratio (L:M) by dividing the total recovered volume of each by the initial dose and expressed as the ratio of percent lactulose recovery to percent mannitol recovery. L:M >0.07 was considered elevated and indicative of EED ${ }^{(15)}$

Anthropometry at ages 15, 18 and 24 months was used for this analysis to examine growth in the 3 months before and 6 months after the assessment of EED and SI biomarkers. LAZ, weight-for-age (WAZ) and weight-for-length (WLZ) $z$ scores relative to the WHO growth standards ${ }^{(1)}$ and prevalence of stunting, underweight and wasting (LAZ, WAZ and WLZ, respectively, $<-2)$ at each interview time point were calculated. Extreme outlying values $(\mid z$ scorel $>6$ ) were omitted from the analysis. Variables for the change in each anthropometric measure from 15 to 18 months and from 18 to 24 months were generated with adjustment for the exact number of days between the pair of measurements. 


\section{Statistical methods}

Biomarkers were log-transformed before analysis. Two orthogonal scores of EED - a 'gut inflammation' (GI) score and a 'gut permeability' (GP) score derived with principal component analysis on log-transformed biomarkers (online Supplementary Table S1) as described previously ${ }^{(24)}$ - were used as indicators of EED along with L:M. The derived EED scores were standardised to units of standard deviation around their mean values and oriented to each have minimum value 0 and higher values indicative of worse intestinal health. Children in the five trial arms were combined for this analysis, as no evidence of supplementation effects on markers of EED or SI was observed ${ }^{(24)}$. Final models were adjusted for supplementation group, as well as for child age and household LSI.

To investigate bivariate associations between biomarkers at 18 months and anthropometry at 18 months and changes in anthropometry over the preceding (15-18 months) and subsequent (18-24 months) age intervals, pairwise Pearson's correlation coefficients were generated for each biomarker anthropometric measure pair.

Longitudinal models were then developed to harmonise associations between biomarkers and anthropometric measures in individual intervals identified using correlation analysis. Mixedeffects (ME) models were developed for repeated measures of LAZ, WLZ and WAZ, an approach that accounts for observed correlations among repeated anthropometric measures of the same child. A spline term was included for age with a knot at 18 months, in accordance with hypotheses about distinct effects of biomarkers on growth trajectories before and after the 18-month assessment. Models were built in a step-by-step manner with each addition evaluated with $P$ values for the added term(s), likelihood ratio tests relative to the simpler model and visual inspection of residual variance plots, as outlined in Grajeda et al. ${ }^{(38)}$. Random slopes, autoregressive correlation structure and correlated random intercepts and slopes were sequentially introduced and evaluated.

Markers of EED (L:M, GI score, GP score) and SI (CRP, AGP) were each added to the longitudinal models as fixed effects with interaction terms between biomarkers and the age and age spline terms to determine the extent to which they explained within- and between-child variation in growth trajectories. Separate models were developed for each biomarker for ease of interpretation given minimal collinearity among biomarkers ${ }^{(24)}$. The models were used to generate coefficients estimating the shift in baseline (15 months) anthropometry and in the rate of growth in anthropometric unit per month from 15 to 18 months and 18 to 24 months, respectively, associated with a 1 -sD change in biomarker value. Confidence intervals for the individual coefficients and $P$ values for the likelihood ratio test comparing otherwise identical models with and without the biomarker were used to evaluate the explanatory value of the biomarker.

The study was conducted according to the guidelines laid down in the Declaration of Helsinki, and all protocols were approved by the institutional review boards of the Johns Hopkins Bloomberg School of Public Health and icddr,b. Written parental consents were required for participation in the supplementation trial and in the EED biomarker assessment. Analyses were conducted using Stata, version 14.1 (StataCorp).

\section{Results}

Of 566 eligible children, parental consent for 539 was obtained for the EED assessment. Households sampled were typical of the setting: all had access to an improved source of water and most ( $83 \%$ ) had an improved sanitation facility, whereas only about $30 \%$ had electricity (Table 1). Most mothers (78\%) had some education, but only $14 \%$ completed high school. Fathers were predominantly employed as farmers (48\%) or day labourers (35\%). Length and weight data were available for 527 (98\%), 539 (100\%) and 513 (95\%) children at the 15-, 18- and 24-month assessments, respectively. Stunting was common, with $45 \%$ stunted at 18 months, and prevalence of underweight and wasting was 37 and 15\%, respectively. L:M, EED scores (GI and GP) and inflammation markers (CRP and AGP) were measured in 476 (88\%), 436 (81\%) and 505 (94\%) children, respectively. Geometric mean of L:M assessed at age 18 months was 0.06 (95\% CI 0.05, 0.06), and 39\% of children had elevated L:M values (>0.07). SI was also common: CRP and AGP were elevated ( $>5 \mathrm{mg} / \mathrm{l}$ and $>1 \mathrm{~g} / \mathrm{l}^{(39)}$, respectively) in 20 and $56 \%$ of children, respectively, but only $4 \%$ had elevated CRP only with normal AGP. The mean values of the standardised GI and GP scores were 2.9 (SD 1.0) and 2.8 (SD 1.0), respectively, with higher values of each indicative of poorer intestinal health $^{(24)}$.

In correlation analysis of biomarkers and anthropometry, few associations were observed between the biomarkers and LAZ (Table 2). In contrast to expectations, L:M was positively correlated with change in LAZ between 15 and 18 months $(\rho=0 \cdot 1$, $P=0.045$ ), such that children with greater L:M at 18 months had grown relatively more in the prior 3 months than those with lower L:M. GP score was positively correlated with LAZ at 18 months $(\rho=0 \cdot 13, P=0 \cdot 005)$, but not with change in length in the surrounding periods. GI score and AGP were not correlated with any LAZ measures. Weight trajectories (WLZ and WAZ) were not correlated with L:M, but were with the other scores, in divergent patterns: GI score was associated with declining WLZ and WAZ from 18 to 24 months only $(\rho=-0 \cdot 13, P=0.006$ and $\rho=-0 \cdot 14, P=0 \cdot 005$, respectively), whereas GP score and AGP were correlated with WLZ and WAZ in all of the intervals examined. GP score was associated with declining WLZ and WAZ from 15 to $18(P \leq 0.01)$ and 18 to 24 months $(P<0.02)$, but was positively correlated with 18 -month WLZ and WAZ $(P<0.04)$. In contrast, higher AGP was associated with lower gains in weight from 15 to 18 months $(P<0.001)$ and somewhat lower WLZ and WAZ $(P=0 \cdot 06)$ at 18 months, but positively correlated with change in WLZ and WAZ from 18 to 24 months $(P<0 \cdot 001)$. In all, correlations with length and weight measures suggested that distinct anthropometric trajectories may have preceded the EED assessment. Correlations between the individual biomarkers that comprise L:M and the GI and GP scores and anthropometric measures further elucidate these trends (online Supplementary Table S2).

Associations of 1-SD increments of EED and SI biomarkers with anthropometric measures at 15 months and growth velocity in $z$ score units per month from $15-18$ and 18-24 months, as determined in ME regression models of 15-24 months trajectories, are shown in Table 3 , along with the statistical 
Table 1. Household and individual characteristics of environmental enteric dysfunction study participants ( $n$ 539) (Numbers and percentages; mean values and standard deviations; geometric means (GM) and $95 \%$ confidence intervals)

\begin{tabular}{|c|c|c|c|}
\hline Characteristics & $n$ & & $\%$ \\
\hline \multicolumn{4}{|l|}{ Household } \\
\hline \multicolumn{4}{|l|}{ Mother's education } \\
\hline None & 116 & & $21 \cdot 6$ \\
\hline $1-9$ years & 348 & & 64.8 \\
\hline Secondary school completed & 73 & & $13 \cdot 6$ \\
\hline \multicolumn{4}{|l|}{ Father's employment } \\
\hline None & 6 & & $1 \cdot 1$ \\
\hline Farmer/owns business & 254 & & 48.2 \\
\hline Day labourer/fisherman & 182 & & 34.5 \\
\hline Private/government service & 85 & & $16 \cdot 1$ \\
\hline Electricity & 165 & & $30 \cdot 7$ \\
\hline Improved water & 537 & & $100 \cdot 0$ \\
\hline Improved toilet ${ }^{\star}$ & 443 & & 82.5 \\
\hline \multicolumn{4}{|l|}{ Child } \\
\hline Sex, female & 269 & & 49.9 \\
\hline Length $(\mathrm{cm})$ & & & \\
\hline Mean & & $76 \cdot 2$ & \\
\hline \multirow{2}{*}{\multicolumn{4}{|c|}{ LAZ }} \\
\hline & & & \\
\hline Mean & & -1.9 & \\
\hline SD & & $1 \cdot 0$ & \\
\hline \multirow{2}{*}{\multicolumn{4}{|c|}{ Weight (kg) }} \\
\hline & & & \\
\hline Mean & & $8 \cdot 8$ & \\
\hline SD & & $1 \cdot 1$ & \\
\hline \multicolumn{4}{|l|}{ WLZ } \\
\hline Mean & & $-1 \cdot 1$ & \\
\hline SD & & 1.0 & \\
\hline \multirow{2}{*}{\multicolumn{4}{|c|}{ WAZ }} \\
\hline & & & \\
\hline Mean & & -1.7 & \\
\hline SD & & 1.0 & \\
\hline WAZ $<-2$ & 197 & & $37 \cdot 1$ \\
\hline \multicolumn{4}{|l|}{ L:M } \\
\hline GM & & 0.06 & \\
\hline $95 \% \mathrm{Cl}$ & & $0.05,0.06$ & \\
\hline \multirow{2}{*}{\multicolumn{4}{|c|}{ MPO (ng/ml) }} \\
\hline & & & \\
\hline GM & & $4460 \cdot 3$ & \\
\hline $95 \% \mathrm{Cl}$ & & $4145 \cdot 0,4799 \cdot 5$ & \\
\hline \multicolumn{4}{|l|}{ AAT $(\mu \mathrm{g} / \mathrm{ml})$} \\
\hline GM & & 326.9 & \\
\hline \multirow{2}{*}{\multicolumn{4}{|c|}{ NEO (nmol/l) }} \\
\hline & & & \\
\hline GM & & 767.4 & \\
\hline $95 \% \mathrm{Cl}$ & & $716 \cdot 5,821 \cdot 8$ & \\
\hline \multicolumn{4}{|l|}{ EndoCAb lgG (MU/ml) } \\
\hline GM & & $45 \cdot 4$ & \\
\hline \multirow{2}{*}{\multicolumn{4}{|c|}{ GLP-2 (ng/ml) }} \\
\hline & & & \\
\hline GM & & $3 \cdot 0$ & \\
\hline $95 \% \mathrm{Cl}$ & & $2 \cdot 9,3 \cdot 1$ & \\
\hline CRP (mg/l) & & & \\
\hline GM & & 1.2 & \\
\hline $95 \% \mathrm{Cl}$ & & $1 \cdot 0,1 \cdot 4$ & \\
\hline $\mathrm{CRP}>5 \mathrm{~g} / \mathrm{l}$ & 103 & & $20 \cdot 4$ \\
\hline AGP $(g / l)$ & & & \\
\hline GM & & 1.0 & \\
\hline $95 \% \mathrm{Cl}$ & & $1 \cdot 0,1 \cdot 1$ & \\
\hline$A G P>1 g / / \S$ & 281 & & $55 \cdot 6$ \\
\hline Gl score & & & \\
\hline Mean & & $2 \cdot 9$ & \\
\hline SD & & 1.0 & \\
\hline GP score & & & \\
\hline Mean & & $2 \cdot 8$ & \\
\hline SD & & 1.0 & \\
\hline
\end{tabular}

LAZ, length-for-age $z$ score; WLZ, weight-for-length $z$ score; WAZ, weight-for-age $z$ score; L:M, lactulose:mannitol ratio; MPO, myeloperoxidase; AAT, $a-1$ antitrypsin; NEO, neopterin; EndoCAb IgG, endotoxin core antibody IgG; GLP-2, glucagon-like peptide-2; CRP, C-reactive protein; AGP, $a-1$ acid glycoprotein; Gl, gut inflammation score; GP, gut permeability score.

* Improved toilet defined as household access to a water-sealed or slab latrine.

$\dagger$ Anthropometry and biomarkers measured at age 18 months.

$\ddagger$ Cutoff for elevated values ${ }^{(15)}$

$\S$ Cutoff for elevated values ${ }^{(39)}$. 
Table 2. Correlation between biomarkers of environmental enteric dysfunction (EED) and systemic inflammation (SI) measured at age 18 months and anthropometric measures at 18 months and their changes from 15 to 18 months and 18 to 24 months of age

\begin{tabular}{|c|c|c|c|c|}
\hline \multirow[b]{2}{*}{ Measures } & \multirow[b]{2}{*}{ Biomarkerł } & \multicolumn{3}{|c|}{ Correlation coefficient $†$} \\
\hline & & $\Delta 15-18$ months & 18 months & $\Delta 18-24$ months \\
\hline \multirow[t]{4}{*}{ LAZ } & L:M & $0 \cdot 10^{* *}$ & -0.04 & -0.07 \\
\hline & Gl score & -0.07 & -0.03 & 0.02 \\
\hline & GP score & 0.04 & $0.13^{\star \star \star}$ & -0.06 \\
\hline & AGP & -0.01 & -0.03 & -0.01 \\
\hline \multirow{4}{*}{ WLZ } & L:M & -0.04 & -0.07 & 0.04 \\
\hline & Gl score & 0.05 & 0.02 & $-0.13^{\star \star *}$ \\
\hline & GP score & $-0 \cdot 13^{\star \star \star}$ & $0 \cdot 10^{\star \star}$ & $-0.12^{\star \star}$ \\
\hline & AGP & $-0.18^{\star \star \star}$ & $-0.08^{\star}$ & $0.19^{\star \star *}$ \\
\hline \multirow[t]{4}{*}{ WAZ } & L:M & 0.03 & -0.06 & -0.02 \\
\hline & Gl score & 0.01 & -0.00 & $-0.14^{\star *}$ \\
\hline & GP score & $-0.12^{\star *}$ & $0.14^{\star \star \star}$ & $-0.14^{\star \star *}$ \\
\hline & AGP & $-0.19^{\star \star *}$ & $-0.08^{\star}$ & $0 \cdot 20^{\star \star *}$ \\
\hline
\end{tabular}

LAZ, length-for-age $z$ score; L:M, lactulose:mannitol ratio; GI, gut inflammation score; GP, gut permeability score; AGP, $a-1$ acid glycoprotein; WLZ, weight-

for-length $z$ score; WAZ, weight-for-age $z$ score.

Statistical significance of the correlation coefficient: * $0.05<P \leq 0.10 ;{ }^{* \star} 0.01<P \leq 0.05 ;{ }^{* \star *} P \leq 0.01$.

$\dagger$ Values are Pearson's correlation coefficients.

$\ddagger$ L:M and AGP were log-transformed before analysis.

Table 3. Associations between environmental enteric dysfunction (EED) and systemic inflammation (SI) biomarkers assessed at age 18 months and repeated anthropometric (anthro.) measures from 15 to 24 months in longitudinal mixed-effects regression models $\dagger$ ( $\beta$-Coefficients with their standard errors)

\begin{tabular}{|c|c|c|c|c|c|c|c|c|c|}
\hline \multirow[b]{2}{*}{ Anthro. measures } & \multirow[b]{2}{*}{ Biomarker } & \multirow[b]{2}{*}{$n+\dagger$} & \multicolumn{2}{|c|}{$\begin{array}{c}\text { 15-month anthro. } \\
\text { difference per 1-sD } \\
\text { difference in biomarkerł } \\
\end{array}$} & \multicolumn{2}{|c|}{$\begin{array}{c}\text { 15-18-month monthly growth } \\
\text { velocity difference per } 1 \text {-SD } \\
\text { difference in biomarker§ } \\
\end{array}$} & \multicolumn{2}{|c|}{$\begin{array}{l}\text { 18-24-month monthly growth } \\
\text { velocity difference per } 1 \text {-sD } \\
\text { difference in biomarkerll }\end{array}$} & \multirow{2}{*}{$\begin{array}{c}P \text { value from likelihood ratio } \\
\text { test for biomarkerł }\end{array}$} \\
\hline & & & $\beta$ & SE & $\beta$ & SE & $\beta$ & SE & \\
\hline \multirow[t]{4}{*}{ LAZ } & L:M & 445 & -0.05 & 0.05 & 0.02 & 0.01 & -0.01 & 0.00 & 0.147 \\
\hline & $\mathrm{Gl}$ & 435 & 0.00 & 0.05 & -0.01 & 0.01 & 0.00 & 0.00 & 0.504 \\
\hline & GP & 435 & $0 \cdot 10^{\star \star}$ & 0.05 & 0.01 & 0.01 & -0.00 & 0.00 & 0.083 \\
\hline & AGP & 504 & -0.05 & 0.05 & -0.00 & 0.01 & -0.00 & 0.00 & 0.698 \\
\hline \multirow[t]{4}{*}{ WLZ } & L:M & 445 & -0.03 & 0.05 & -0.01 & 0.01 & 0.00 & 0.00 & 0.613 \\
\hline & Gl & 435 & -0.02 & 0.05 & 0.01 & 0.01 & $-0.01^{\star \star \star}$ & 0.00 & 0.024 \\
\hline & GP & 435 & $0.16^{\star \star \star}$ & 0.05 & $-0.03^{\star \star \star}$ & 0.01 & $-0.01^{\star *}$ & 0.00 & 0.000 \\
\hline & AGP & 504 & 0.02 & 0.04 & $-0.04^{\star \star \star}$ & 0.01 & $0.02^{\star \star *}$ & 0.00 & 0.000 \\
\hline \multirow[t]{4}{*}{ WAZ } & L:M & 445 & -0.05 & 0.05 & 0.00 & 0.01 & -0.00 & 0.00 & 0.727 \\
\hline & Gl & 435 & -0.01 & 0.05 & 0.00 & 0.01 & $-0.01^{\star \star \star}$ & 0.00 & 0.019 \\
\hline & GP & 435 & $0.17^{\star \star \star}$ & 0.05 & $-0.02^{\star \star}$ & 0.01 & $-0.01^{\star \star \star}$ & 0.00 & 0.000 \\
\hline & AGP & 504 & -0.01 & 0.04 & $-0.03^{\star \star \star}$ & 0.01 & $0.02^{\star \star \star}$ & 0.00 & 0.000 \\
\hline
\end{tabular}

LAZ, length-for-age $z$ score; L:M, lactulose:mannitol ratio; GI, gut inflammation score; GP, gut permeability score; AGP, $a-1$ acid glycoprotein; WLZ, weight-for-length $z$ score; WAZ, weight-for-age $z$ score; LSI, living standards index.

Significance of the individual coefficient in the mixed-effects model: ${ }^{* *} 0.01<P \leq 0.05 ; * \star * ~ P \leq 0.01$. Asterisks are not displayed if the overall likelihood ratio test $P$ value is $>0.10$.

† Values are coefficients and standard errors from mixed-effects linear regression models with a continuous biomarker term, continuous age term, spline term for age after 18 months, interactions between the continuous biomarker and the age and age spline terms, and random intercepts and random slopes for each child. Models were developed separately for each biomarker. The LAZ models were adjusted for child sex, dichotomous LSI and interactions between LSI and age spline terms, and assigned supplementation group. WAZ and WLZ models were adjusted for sex, dichotomous LSI and supplementation group.

¥ Coefficient for continuous biomarker term in mixed-effects model, which indicates shift in baseline (15 months) anthropometric measure with each standard deviation change in biomarker value.

$\S$ Coefficient for interaction term for continuous biomarker by continuous age interaction in mixed-effects model. This term indicates the shift in the monthly rate of change in the anthropometric measure with age before 18 months for each standard deviation change in biomarker value.

॥ Coefficient for interaction term for continuous biomarker by age spline interaction in mixed-effects model. This term indicates the shift in the monthly rate of change in the anthropometric measure with age after 18 months for each standard deviation change in biomarker value.

I L:M and AGP were log-transformed, and all biomarkers and scores were centred at their mean values and standardised to standard deviation units.

†† Participants with complete data for the model variables were included in each model.

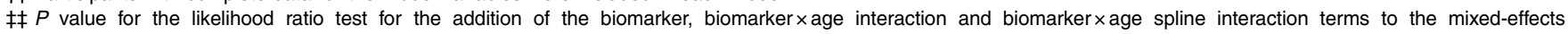
regression model.

significance of the biomarker's contribution to each model according to the likelihood ratio test. Longitudinal models included all children with complete data for the relevant variables, and thus sample sizes differed slightly between models to maximise the sample size in each. Overall, only GP score was associated with LAZ trajectory, and its contribution to the model was marginal (likelihood ratio test $P=0 \cdot 08$ ). Children with a 1- SD greater GP score tended to have 0.1 greater LAZ at 15 months, corresponding to $0 \cdot 28$ - $\mathrm{cm}$ greater length, although their rate of growth from 15 to 24 months was not different from those with lower GP score. Initial WLZ and WAZ at 15 months were greater by $0.16 z$ (SE 0.05) and $0.17 z$ (SE 0.05), 
respectively, in those with GP score 1 sD greater than the mean, corresponding to $0 \cdot 16 \mathrm{~kg}$ higher weight at 15 months. None of the other biomarkers was associated with differences in 15-month WLZ or WAZ. GP score and AGP were associated with small but highly significant decreases in rate of WLZ and WAZ change from 15 to 18 months $(\beta-0.03$ (se 0.01 ) and $\beta-0.02$ (sE 0.01$) z$ score units/month, respectively, for GP score and $\beta-0.04$ (SE 0.01) and $\beta-0.03$ (SE 0.01) $z$ score units/month, respectively, for AGP). These coefficients correspond to $0.04 \mathrm{~kg}$ less weight gained over the 15-18-month period in children with GP score 1- SD greater than the mean and $0.08 \mathrm{~kg}$ less weight gained in the same interval in children with 1- SD greater AGP. In the 18-24-month period, GI score and GP score were negatively associated with change in WLZ and WAZ $(\beta-0 \cdot 01$ (SE 0.00) for all), whereas AGP was positively associated with WLZ and WAZ ( $\beta 0.02$ (sE 0.00) for both). A 1- sD greater GI or GP score was associated with $0.06 \mathrm{~kg}$ less weight gain over the 6 months from 18 to 24 months, whereas a 1- sD greater AGP value was associated with $0.09 \mathrm{~kg}$ more weight gained between 18 and 24 months.

In ME models for individual EED biomarkers, of the GI score markers, only AAT was negatively associated with change in WLZ and WAZ between 18 and 24 months (online Supplementary Table S3). Of the GP score markers, GLP-2 was strongly inversely associated with 15-month length and positively associated with 18-24-month change in WLZ and WAZ, whereas EndoCAb, with a small but highly significant inverse association with 15-18-month change in WLZ and WAZ, contributed more to GP score associations with 15-18-month weight gain velocity. EndoCAb was associated with greater 15 months WLZ and WAZ, whereas GLP-2 was associated with lower 15-month WLZ and WAZ, directionality that is concordant given their opposite loadings on the GP score. Associations with CRP were examined but none was observed in simple or ME regression models (results not shown).

\section{Discussion}

Among Bangladeshi children participating in a trial of CFS, EED and SI, comprehensively assessed with a panel of biomarkers at age 18 months, were associated with children's weight-gain trajectories but not their linear growth in the 2nd year of life. The lack of evidence for an EED impact on linear growth occurred despite a high prevalence of stunting in this cohort, an extensive burden of EED and SI assessed with a panel of accepted and novel biomarkers, and the use of longitudinal models to comprehensively characterise growth trajectories from 15 to 24 months of age. Our findings contrast with several studies that have reported associations between EED and linear growth $^{(17-20,22,23,40,41)}$, but observed links to weight gain suggest that effects may manifest in important health and development outcomes aside from restricted linear growth.

An advantage of our study design is having growth data that precede the EED measurement. This is particularly important for examining EED in the 2nd year of life, as during this period linear growth rates typically slow down compared with greatest growth velocity in infancy, yet smaller children may grow relatively more rapidly than children of a healthy size to recover from prior faltering ${ }^{(6,42)}$. These characteristics of linear growth highlight the importance of understanding and accounting for both usual growth dynamics and individual growth history when examining the impact of EED on growth. Our longitudinal models used length measured at multiple time points surrounding the EED assessment to compare growth velocity from 18-24 months among otherwise similar children, including with respect to their initial size at 15 months and growth in the 15- to 18-month interval.

The null association between EED and linear growth differs from findings in several other studies ${ }^{(17-20,22,23,40,41)}$, although it is not unique to our study ${ }^{(22,43,44)}$. Several factors could contribute to this finding. EED may be relatively transient ${ }^{(45)}$, such that children with elevated markers at this single time point were not necessarily more burdened with EED over the preceding or subsequent months. A recent publication describing high within-child variability in faecal EED biomarkers over the first 2 years of life corroborates the hypothesis that EED is more transient than chronic in young children $^{(46)}$. Assuming, then, that EED assessed at 18 months is indicative of present status rather than prior or sustained illness, an effect on growth would most likely be observed in the subsequent months. However, the timing of assessments in the 2nd year of life may have precluded observing prospective associations with growth, as previous reports of associations between EED and subsequent growth have largely been observed in children $<15$ months old ${ }^{(17,18,20,40,41)}$. Additionally, the effect of EED at a single time point may be insufficient to measurably alter linear growth in a subsequent 6-month interval, similarly observed by Arndt et al. ${ }^{(22)}$ and Kosek et $a l .{ }^{(44)}$ in a multi-country study. Finally, the association of EED with WLZ that we observed could precede length effects that manifest beyond the available 6-month follow-up, as WLZ changes can portend future $\mathrm{LAZ}^{(47)}$, with effects on LAZ slow to emerge amidst slowing linear growth rates.

In contrast to length, however, weight gain trajectory was significantly perturbed by EED and SI. Children with elevated AGP gained relatively less weight from 15 to 18 months but seemed to recover from 18 to 24 months, whereas those with elevated GI score gained less weight from 18 to 24 months and those with elevated GP score gained less weight in both intervals. This temporal divergence is consistent with AGP marking the end of an infection followed by catch-up growth rather than indicating sustained subclinical infection, whereas GI and GP scores may indicate current or ongoing conditions that impair subsequent weight gain. Observed WLZ declines could represent impaired muscle or fat deposition, which could precede length growth effects that are slower to emerge. Alternately, short-term WLZ fluctuations could provide some cushion that shields linear growth from mild, transient insults. Previous studies investigating EED and severe acute malnutrition (SAM), defined by WLZ, have reported inverse associations between EED and WLZ at presentation and during recovery with therapeutic feeding ${ }^{(15,48)}$, although older studies often conflated symptomatic diarrhoeal illness with subclinical $\mathrm{EED}^{(48)}$. Before this paper, there has been little evidence to show an association of EED with WLZ variation that does not reach the severity of SAM. Still, one new multi-site study reported a similar set of findings to ours, with stronger 
associations of EED and SI with WLZ than LAZ changes in repeated assessments over the first 2 years of life ${ }^{(44)}$.

In this study of EED nested within an RCT, an overarching hypothesised pathway from CFS to growth via improved EED was not supported by our findings. In the parent trial, groups assigned to receive CSF had 0.07-0.10 greater mean LAZ and 5-6 percentage point reduction in stunting prevalence at 18 months of age following a year of supplementation relative to an un-supplemented control group ${ }^{(25)}$. We found minimal associations, however, between EED and linear growth, which suggests, in combination with previously reported null effects of the CFS on EED ${ }^{(24)}$, that food supplementation affected growth via pathways not mediated by EED. This may be due primarily to a lack of benefit for EED of the particular CFS formulations or the quantities given, or because EED was not a major factor limiting growth in this setting, possibilities that require further investigation to inform future CFS interventions. Supplementation, EED and growth could, alternately, be associated such that supplementation blunts the impact of EED on growth. This could explain the null associations we observe between EED and linear growth, as $4 / 5$ of the participants had received CFS. Analyses stratified by supplementation group did not suggest effect modification of that nature, although the study was not powered to address that possibility.

The strengths of the present study include the very large sample size, comprehensive panel of EED and SI biomarkers including the widely used L:M test, and repeated high-quality anthropometry assessments. Weaknesses include having EED measured only once. The timing of the EED assessment was driven by a primary hypothesis focused on the effects of the supplementation trial on EED markers, which necessitated assessment of EED in the middle of the 2nd year of life, and supported prioritising a larger sample size over repeated EED assessments. The lack of a true gold standard test of EED inhibits the interpretation of the biomarkers where they diverge from L:M, which is a limitation in this field of study more generally.

In this rural Bangladesh cohort where stunting and EED were common, EED exerted little influence over linear growth trajectories during the 2nd year of life, whereas weight gain was relatively more compromised by EED and SI. The high prevalence of EED and SI that we observed in this study along with effects on subsequent weight gain distinct from gains in length suggest potential consequences of EED on body composition, the impact of which may be the subject of future study as we continue to investigate strategies to support optimal growth.

\section{Acknowledgements}

The authors thank Margia Arguello, Sarah Baker and Afreen Zaman Khan for assistance with laboratory measures, and John McGready for help with statistical model development.

This work was supported by the United States Department of Agriculture (USDA) National Institute of Food and Agriculture's Food Aid Nutrition Enhancement Program (2010-38418-21732); National Institutes of Health (NIH) (R21HD081503); and Johns Hopkins Sight and Life Global Nutrition Research Institute.
USDA, NIH and Johns Hopkins Sight and Life Global Nutrition Research Institute had no role in the design, analysis or writing of this article.

R. K. C., K. J. S. and P. C. designed research; R. K. C., K. J. S., S. S., R. R., H. A., S. M., K. P. W. and P. C. conducted research; R. K. C. analysed data and wrote the paper; K. J. S. and P. C. made substantial contributions to data analysis and manuscript revisions; and all authors read and approved the final manuscript.

None of the authors has any conflicts of interest to declare.

\section{Supplementary material}

For supplementary material/s referred to in this article, please visit https://doi.org/10.1017/S0007114517003683

\section{References}

1. World Health Organization Multicentre Growth Reference Study Group (2006) WHO child growth standards based on length/ height, weight and age. Acta Paediatr Suppl 450, 76-85.

2. Headey DD (2013) Developmental drivers of nutritional change: a cross-country analysis. World Dev 42, 76-88.

3. Black RE, Allen LH, Bhutta ZA, et al. (2008) Maternal and child undernutrition: global and regional exposures and health consequences. Lancet 371, 243-260.

4. Victora CG, Adair L, Fall C, et al. (2008) Maternal and child undernutrition: consequences for adult health and human capital. Lancet 371, 340-357.

5. Dewey KG \& Begum K (2011) Long-term consequences of stunting in early life. Matern Child Nutr 7, 5-18.

6. Victora CG, de Onis M, Hallal PC, et al. (2010) Worldwide timing of growth faltering: revisiting implications for interventions. Pediatrics 125, e473-e480.

7. United Nations Children's Fund (2013) Improving Child Nutrition: the Achievable Imperative for Global Progress. New York: United Nations Children's Fund.

8. International Food Policy Research Institute (2015) Global Nutrition Report 2015: Actions and Accountability to Advance Nutrition and Sustainable Development. Washington, DC: International Food Policy Research Institute.

9. Humphrey JH (2009) Child undernutrition, tropical enteropathy, toilets, and handwashing. Lancet 374, 1032-1035.

10. Korpe PS \& Petri WA Jr (2012) Environmental enteropathy: critical implications of a poorly understood condition. Trends Mol Med 18, 328-336.

11. Mapesa JO, Maxwell AL \& Ryan EP (2016) An exposome perspective on environmental enteric dysfunction. Environ Health Perspect 124, 1121-1126.

12. Crane RJ, Jones KD \& Berkley JA (2015) Environmental enteric dysfunction: an overview. Food Nutr Bull 36, S76-S87.

13. Guerrant RL, Oria RB, Moore SR, et al. (2008) Malnutrition as an enteric infectious disease with long-term effects on child development. Nutr Rev 66, 487-505.

14. Goto R, Panter-Brick C, Northrop-Clewes CA, et al. (2002) Poor intestinal permeability in mildly stunted Nepali children: associations with weaning practices and Giardia lamblia infection. Br J Nutr 88, 141-149.

15. Hossain MI, Nahar B, Hamadani JD, et al. (2010) Intestinal mucosal permeability of severely underweight and nonmalnourished Bangladeshi children and effects of nutritional rehabilitation. I Pediatr Gastroenterol Nutr 51, 638-644. 
16. Manary MJ, Abrams SA, Griffin IJ, et al. (2010) Perturbed zinc homeostasis in rural 3-5-y-old Malawian children is associated with abnormalities in intestinal permeability attributed to tropical enteropathy. Pediatr Res 67, 671-675.

17. Lunn PG, Northrop-Clewes CA \& Downes RM (1991) Intestinal permeability, mucosal injury, and growth faltering in Gambian infants. Lancet 338, 907-910.

18. Kosek M, Haque R, Lima A, et al. (2013) Fecal markers of intestinal inflammation and permeability associated with the subsequent acquisition of linear growth deficits in infants. $\mathrm{Am}$ J Trop Med Hyg 88, 390-396.

19. Weisz AJ, Manary MJ, Stephenson K, et al. (2012) Abnormal gut integrity is associated with reduced linear growth in rural Malawian children. J Pediatr Gastroenterol Nutr 55 , 747-750.

20. Campbell DI, Elia M \& Lunn PG (2003) Growth faltering in rural Gambian infants is associated with impaired small intestinal barrier function, leading to endotoxemia and systemic inflammation. J Nutr 133, 1332-1338.

21. Mondal D, Minak J, Alam M, et al. (2012) Contribution of enteric infection, altered intestinal barrier function, and maternal malnutrition to infant malnutrition in Bangladesh. Clin Infect Dis 54, 185-192.

22. Arndt MB, Richardson BA, Ahmed T, et al. (2016) Fecal markers of environmental enteropathy and subsequent growth in Bangladeshi children. Am J Trop Med Hyg 95, 694-701.

23. Guerrant RL, Leite AM, Pinkerton R, et al. (2016) Biomarkers of environmental enteropathy, inflammation, stunting, and impaired growth in children in Northeast Brazil. PLOS ONE 11, e0158772.

24. Campbell RK, Schulze K, Shaikh S, et al. (2017) Biomarkers of environmental enteric dysfunction among children in rural Bangladesh. J Pediatr Gastroenterol Nutr 65, 40-46.

25. Christian P, Shaikh S, Shamim AA, et al. (2015) Effect of fortified complementary food supplementation on child growth in rural Bangladesh: a cluster-randomized trial. Int J Epidemiol 44, 1862-1876.

26. West KP Jr., Christian P, Labrique AB, et al. (2011) Effects of vitamin A or beta carotene supplementation on pregnancyrelated mortality and infant mortality in rural Bangladesh. JAMA 305, 1986-1995.

27. West KP Jr., Shamim AA, Mehra S, et al. (2014) Effect of maternal multiple micronutrient vs iron-folic acid supplementation on infant mortality and adverse birth outcomes in rural Bangladesh: the JiVitA-3 randomized trial. JAMA $\mathbf{3 1 2}$, 2649-2658.

28. Klemm RD, Labrique AB, Christian P, et al. (2008) Newborn vitamin A supplementation reduced infant mortality in rural Bangladesh. Pediatrics 122, e242-e250.

29. Shamim AA, Kabir A, Merrill RD, et al. (2013) Plasma zinc, vitamin $\mathrm{B}(12)$ and alpha-tocopherol are positively and plasma gamma-tocopherol is negatively associated with $\mathrm{Hb}$ concentration in early pregnancy in north-west Bangladesh. Public Health Nutr 16, 1354-1361.

30. Merrill RD, Shamim AA, Ali H, et al. (2012) High prevalence of anemia with lack of iron deficiency among women in rural Bangladesh: a role for thalassemia and iron in groundwater. Asia Pac J Clin Nutr 21, 416-424.

31. Sikder SS, Labrique AB, Craig IM, et al. (2015) Patterns and determinants of care seeking for obstetric complications in rural northwest Bangladesh: analysis from a prospective cohort study. BMC Health Serv Res 15, 166.
32. Rah JH, Shamim AA, Arju UT, et al. (2010) Difference in ponderal growth and body composition among pregnant vs. never-pregnant adolescents varies by birth outcomes. Matern Child Nutr 6, 27-37.

33. Dabritz J, Musci J \& Foell D (2014) Diagnostic utility of faecal biomarkers in patients with irritable bowel syndrome. World J Gastroenterol 20, 363-375.

34. Campbell DI, McPhail G, Lunn PG, et al. (2004) Intestinal inflammation measured by fecal neopterin in Gambian children with enteropathy: association with growth failure, Giardia lamblia, and intestinal permeability. J Pediatr Gastroenterol Nutr 39, $153-157$.

35. Crossley JR \& Elliott RB (1977) Simple method for diagnosing protein-losing enteropathies. Br Med J 1, 428-429.

36. Rowland KJ \& Brubaker PL (2011) The 'cryptic' mechanism of action of glucagon-like peptide-2. Am J Physiol Gastrointest Liver Physiol 301, G1-G8.

37. Gunnsteinsson S, Labrique AB, West KP Jr., et al. (2010) Constructing indices of rural living standards in Northwestern Bangladesh. J Health Popul Nutr 28, 509-519.

38. Grajeda LM, Ivanescu A, Saito M, et al. (2016) Modelling subject-specific childhood growth using linear mixed-effect models with cubic regression splines. Emerg Themes Epidemiol 13, 1 .

39. Thurnham DI, Northrop-Clewes CA \& Knowles J (2015) The use of adjustment factors to address the impact of inflammation on vitamin A and iron status in humans. J Nutr $\mathbf{1 4 5}$, 1137S-1143S.

40. Panter-Brick C, Lunn PG, Langford RM, et al. (2009) Pathways leading to early growth faltering: an investigation into the importance of mucosal damage and immunostimulation in different socio-economic groups in Nepal. Br J Nutr 101, $558-567$.

41. Goto R, Mascie-Taylor CG \& Lunn PG (2009) Impact of intestinal permeability, inflammation status and parasitic infections on infant growth faltering in rural Bangladesh. $\mathrm{BrJ}$ Nutr 101, 1509-1516.

42. Richard SA, Black RE, Gilman RH, et al. (2014) Catch-up growth occurs after diarrhea in early childhood. J Nutr 144, 965-971.

43. Denno DM, VanBuskirk K, Nelson ZC, et al. (2014) Use of the lactulose to mannitol ratio to evaluate childhood environmental enteric dysfunction: a systematic review. Clin Infect Dis 59, S213-S219.

44. Kosek M, Ahmed T, Bhutta Z, et al. (2017) Causal pathways from enteropathogens to environmental enteropathy: findings from the MAL-ED birth cohort study. EBioMedicine 18, 109-117.

45. Lee GO, McCormick BJJ, Seidman JC, et al. (2017) Infant nutritional status, feeding practices, enteropathogen exposure, socioeconomic status, and illness are associated with gut barrier function as assessed by the lactulose mannitol test in the MAL-ED birth cohort. Am J Trop Med Hyg 97, 281-290.

46. McCormick BJ, Lee GO, Seidman JC, et al. (2017) Dynamics and trends in fecal biomarkers of gut function in children from 1-24 months in the MAL-ED study. Am J Trop Med Hyg 96, 465-472.

47. Richard SA, Black RE, Gilman RH, et al. (2012) Wasting is associated with stunting in early childhood. J Nutr $\mathbf{1 4 2}$, $1291-1296$

48. Behrens RH, Lunn PG, Northrop CA, et al. (1987) Factors affecting the integrity of the intestinal mucosa of Gambian children. Am J Clin Nutr 45, 1433-1441. 